\title{
Geometria Dinâmica e Método Clínico de Piaget: visando o pensamento geométrico das transformações
}

\author{
Larissa Weyh Monzon - PPGIE/UFRGS - larissamonzon@ gmail.com \\ Marcus Vinicius de Azevedo Basso - PPGIE/UFRGS - mbasso@ufrgs.br
}

\begin{abstract}
Resumo: Nesse artigo analisa-se o papel do uso de recursos digitais de Geometria Dinâmica em entrevista baseada no Método Clínico de Jean Piaget considerando o estudo do pensamento geométrico em relação a transformações no plano. Para esta análise desenvolveu-se uma sequência de atividades que contempla transformações geométricas utilizando o software GeoGebra em uma entrevista clínica com dez estudantes do Ensino Básico. O uso desse recurso proporcionou aos sujeitos da entrevista uma forma de interação e manipulação para construir conceitos, como também, para o pesquisador entender o pensamento dos entrevistados. Os dados apontam para a possibilidade de integração do Método Clínico e uso de mídias digitais possibilitando a ação do sujeito sobre objetos de geometria.
\end{abstract}

Palavras-chave: Geometria dinâmica, Método Clínico, Piaget, transformações geométricas

\section{Dynamic Geometry and Clinical Method of Piaget: aiming at the geometric thinking of transformations}

\begin{abstract}
This article analyses the role of the use of digital resources of Dynamic Geometry in interview based on Jean Piaget Clinical Method, considering the study of geometrical thought related to transformations of the plan. For this analysis was developed a sequence of activities who includes geometrical transformations using the software GeoGebra in a clinical interview with ten students of basic education. The use of this resource provided the subjects of the interview with a form of interaction and manipulation to construct concepts, as well as for the researcher to understand the thoughts of the interviewees. The data point to the possibility of integration of the Clinical Method and the use of digital medias making possible the action of the subject on geometry objects.
\end{abstract}

Key words: Dynamic geometry, Clinical Method, Piaget, geometric transformations

\section{Introdução}

Em relação à Matemática, Tall (1998) diz que as tecnologias realçam a diferença entre as habilidades matemáticas padronizadas e o pensar matematicamente. As máquinas estão fazendo as contas o que significa que a matemática que envolve habilidades operatórias não é mais tão necessária para o cidadão atual. É necessário que nossos alunos apreciem a matemática para que consigam explorar, analisar e compreender os diferentes conhecimentos e raciocínios que esta ciência pode propiciar.

Para o pensar matemática hoje dispomos de diversos recursos digitais e podemos associá-las a ferramentas interativas que disponibilizam objetos dinâmicos e manipuláveis, os quais estão se refletindo de forma positiva em pesquisas da Educação Matemática (GRAVINA, 1996; GRAVINA e BASSO, 2012). Tais recursos compõem o que chamamos de Matemática Dinâmica. 
Já o estudo dos conceitos e propriedades geométricas podem exigir um pensamento lógico e habilidades mentais superiores às padronizadas e pré-definidas do uso de cálculo e aplicação de fórmulas e, com isso, problemas geométricos bem elaborados possibilitam o pensar e fazer matemática. Para isso, temos disponíveis recursos de Matemática Dinâmica e aqui, especificamente, de Geometria Dinâmica. Tais recursos disponibilizam a manipulação dos seus elementos propiciando, portanto, a ação do sujeito sobre os objetos digitais.

Diante disso, este artigo apresenta um estudo sobre a utilização de recursos digitais da Geometria Dinâmica como um instrumento de experiência colocado diante de um sujeito para estudar seu pensamento referente às transformações geométricas no plano, baseado no Método Clínico de Piaget.

Assim esse estudo apresenta-se organizado da seguinte maneira: na seção 2 há uma análise das potencialidades tecnológicas para a matemática; a seção 3 apresenta um breve estudo sobre o Método Clínico; a seção 4 contempla as transformações geométricas e sua importância para o pensamento matemático; e finalmente as últimas seções apresentam a análise de uma experiência sobre as transformações geométricas utilizando a Geometria Dinâmica com questionamentos do pesquisador baseados no Método Clínico de Piaget.

\section{A Matemática Dinâmica}

Para Basso e Notare (2015) as tecnologias e os ambientes dinâmicos proporcionam a evolução da Matemática como também da Educação Matemática, abrindo novas possibilidades para o pensamento matemático. Essas ferramentas não são vistas como recurso atrativo ou apenas de praticidade aos estudantes, mas sim como possibilidades de construção de conceitos e raciocínios em matemática.

A matemática surgiu da manipulação de objetos (TALL, 1998), e utilizar Matemática Dinâmica no ensino pode proporcionar aos estudantes a manipulação dos objetos matemáticos estudados via mídias digitais. Essas colocam à disposição do ensino de matemática diversas ferramentas interativas que oferecem ao usuário a visualização, o dinamismo e a manipulação de objetos matemáticos até então não acessíveis, permitindo construir modelos mentais e desenvolver habilidades de generalizações. Para os autores Basso e Notare (2015), há níveis mais elevados de ideias matemáticas que não tem suporte nos modelos físicos e, nestes casos, os computadores podem fornecer a possibilidade de manipulação desses elementos matemáticos.

Mais especificamente em geometria, é essencial, para uma construção adequada da noção do objeto geométrico, a relação das suas propriedades com sua representação. Os livros didáticos de matemática trazem essa relação, mas com desenhos particulares de cada objeto matemático estudado. Para um professor, na sala de aula, fica inviável ou mesmo impossível produzir a variedade de representações necessárias para que os estudantes construam e estabeleçam relações entre os conceitos geométricos.

Essas mídias digitais privilegiam o pensamento visual. Esse movimento, a possibilidade de arrastar, faz com que os alunos explorem, tentem, aguçando muitas vezes sua curiosidade diante das diferentes características envolvidas e fazem com que os alunos reflitam sobre os resultados obtidos após cada movimento. A possibilidade de manipulação de elementos matemáticos pelos sujeitos proporcionada pelas mídias digitais pode ser articulada com as a utilização do Método Clínico de Piaget, o que será discutido nas próximas seções. 


\section{Método Clínico de Jean Piaget}

O objetivo do Método Clínico de Jean Piaget, segundo Delval (2002), é descobrir o pensamento que o sujeito não tem explícito, se constituindo em uma sucessão de intervenções do experimentador em resposta a cada ação do sujeito. É um procedimento de coleta e análise de dados para estudar o pensamento do sujeito mediante entrevistas abertas, que podem vir acompanhadas de uma experiência e que buscam compreender o curso de pensamento do entrevistado.

No desenvolvimento dos estudos de Piaget houve a necessidade de criar experiências físicas para gerar situações nas quais era preciso colocar em ação o pensamento do sujeito em relação à determinada operação que pode ou não ter sido interiorizada. Assim, tratando-se de problemas concretos e de situações práticas, Piaget se apoia em um material, na ação do sujeito e o que o sujeito diz.

O método clínico é um procedimento para investigar como as crianças pensam, percebem, agem e sentem, que procura descobrir o que não é evidente no que os sujeitos fazem ou dizem, o que está por trás da aparência de sua conduta, seja em ações ou palavras (DELVAL, 2002, p. 67).

O autor explica que há três tipos de utilização do método clínico: a entrevista livre (sem material), explicação de uma situação que seria transformações de um material e as ações sobre a realidade. Nas entrevistas que utilizam o Método Clínico, o sujeito é colocado diante de transformações de objetos e as ações que o sujeito pratica e suas explicações nos informam sobre as propriedades que atribui à realidade. Para criar uma experiência, o pesquisador deve partir de algumas hipóteses acerca das razões pelas quais se produz um determinado fenômeno e cria uma situação experimental para comprovar se essas hipóteses estão corretas.

Para pesquisar sobre o pensamento geométrico em relação a diferentes situações, onde há muitas variáveis, no qual um objeto pode ser transformado de diferentes maneiras, podemos utilizar a Geometria Dinâmica. Dessa forma, os recursos digitais podem servir como um recurso de uma experiência para criar situações onde colocamos um sujeito em ação diante do conhecimento a ser investigado.

Piaget ao verificar sobre situações concretas da realidade utilizava materiais manipulativos. Atualmente, podemos não só verificar o pensamento concreto do sujeito em relação ao pensamento matemático como também suas diferentes generalizações mentais que os recursos tecnológicos podem proporcionar.

Na próxima seção apresenta-se o estudo sobre as transformações geométricas e comportamento do pensamento matemático esperado para situações envolvendo esses conceitos matemáticos.

\section{0 pensamento geométrico e as transformações}

Segundo os Parâmetros Curriculares Nacionais (PCN) o ensino de Matemática deve contemplar o estudo do Espaço e das Formas, pois por meio dele "o aluno desenvolve um tipo especial de pensamento que lhe permite compreender, descrever e representar, de forma organizada, o mundo em que vive" (BRASIL, 1998, p. 49).

Especificamente sobre as transformações geométricas, os PCN mencionam:

Deve destacar-se também nesse trabalho a importância das transformações geométricas (isometrias, homotetias), de modo que permita o desenvolvimento de habilidades de percepção espacial e como recurso para induzir de forma experimental a descoberta, por exemplo, das condições para que duas figuras sejam congruentes ou semelhantes. (BRASIL, 1998, p. 51) 
Como referência mais atual, temos a Base Nacional Comum Curricular (BNCC) homologada em dezembro de 2017, nela o ensino de geometria está presente desde a Educação Infantil percorrendo todo o Ensino Básico. Em relação as transformações de figuras o documento menciona que no $4^{\circ}$ do ensino fundamental o aluno deve reconhecer a simetria de reflexão em figuras e em pares de figuras, no $5^{\circ}$ e $6^{\circ}$ ano o reconhecimento da congruência em reduções e ampliações. No $7^{\circ}$ e $8^{\circ}$ ano as transformações são ainda mais valorizadas, o documento sugere que as transformações geométricas de translação, rotação e reflexão dos polígonos no plano cartesiano podem ser ensinadas fazendo uso de software de geometria dinâmica.

O pensamento geométrico deve abranger a interpretação de localização e deslocamento de elementos no plano, reconhecendo direções e sentidos, estabelecer relações entre as figuras desenvolvendo conceitos de transformação, ampliação e redução.

As transformações geométricas são funções que associam cada ponto do plano em um outro ponto, também do plano, segundo uma regra. As transformações que preservam os segmentos, os ângulos e as medidas são as isometrias, ou seja, transformam uma figura em outra congruente.

Segundo Medeiros (2012) a translação está relacionada com o conceito de vetor, que significa transportar. Um vetor é dado pela direção, sentido e comprimento. Ao que segue temos um exemplo de transformação do triângulo $\mathrm{ABC}$ em outro triângulo A'B'C' por um vetor, ou seja, uma translação:
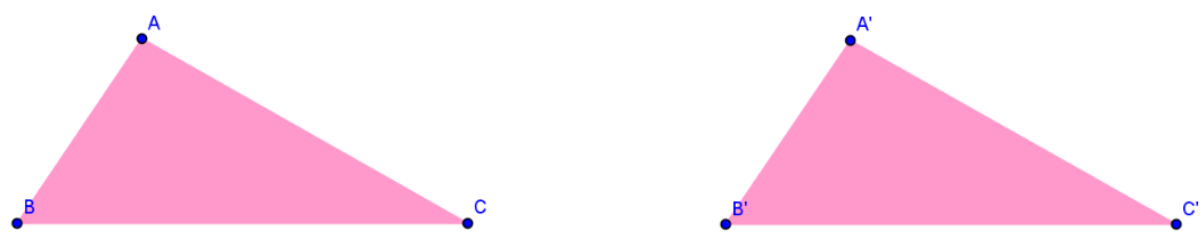

Figura 1. Exemplo de translação

A reflexão em relação à uma reta nos dá duas figuras simétricas, uma imagem espelhada da outra em relação à reta considerada, chamada de eixo de simetria. Por exemplo, na Figura 2, temos um triângulo $\mathrm{ABC}$ onde os pontos A'B'C' estão em lado oposto à reta, mas a uma mesma distância do eixo de simetria.

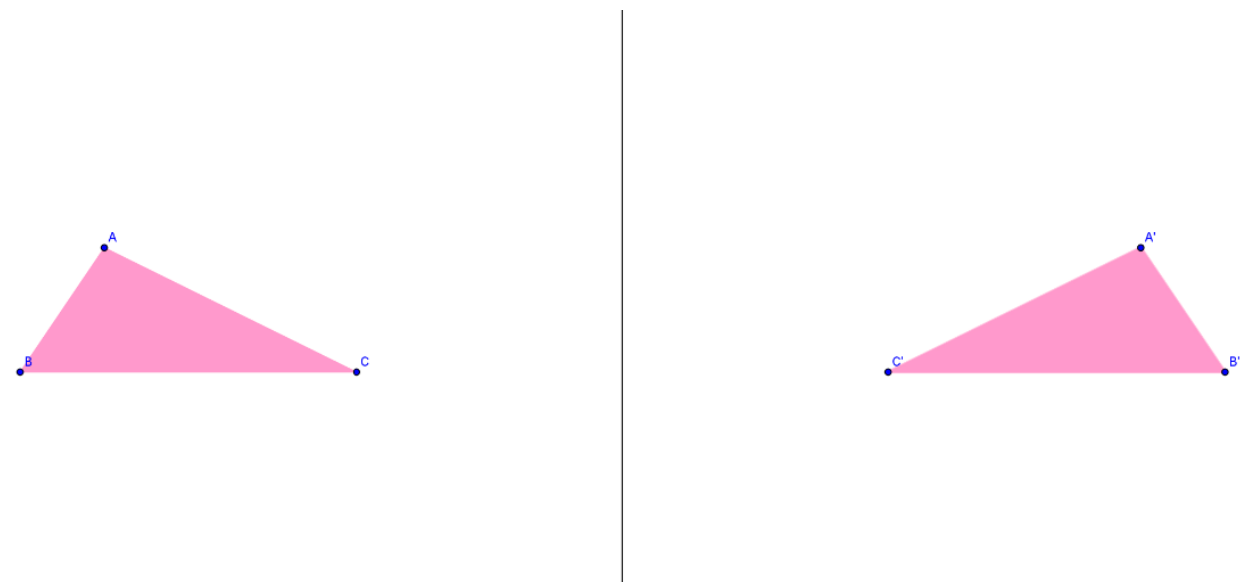

Figura 2. Exemplo de reflexão em relação à reta 
A reflexão em relação a um ponto, por exemplo, de um ponto $\mathrm{P}$ em relação ao ponto A dá origem ao ponto $\mathrm{P}$ ' onde A é o ponto médio do segmento PP' (STORMOWSKI, 2008). Para entender essa reflexão tratando-se de uma figura, verificase essa transformação para cada vértice a ser refletido, ao que segue na Figura 3, temos essa transformação do triângulo $\mathrm{ABC}$ (onde $\mathrm{C}=\mathrm{C}^{\prime}$ ) em relação ao ponto $\mathrm{C}$ (seu próprio vértice) originando o triângulo $A^{\prime} B^{\prime} C^{\prime}$, no qual observa-se que o ponto $C^{\prime}$ é o ponto médio do segmento AA' e do segmento BB'.

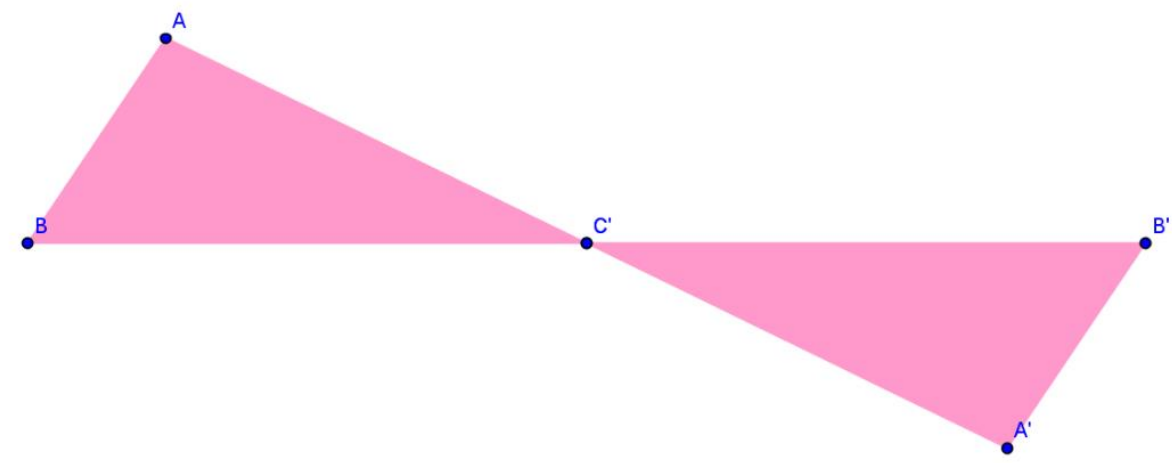

Figura 3. Exemplo de reflexão em relação a um ponto

Os três exemplos de transformações isométricas descritas acima são as transformações estudadas na investigação descrita a seguir. As figuras apresentadas foram geradas com a própria ferramenta utilizada na entrevista com os sujeitos da pesquisa.

\section{Metodologia: a entrevista clínica}

Para verificar a possibilidade do uso de recursos digitais em relação ao estudo do pensamento geométrico utilizando o Método Clínico de Piaget foi elaborada uma sequência de atividades que exploravam as transformações geométricas utilizando o software de Matemática Dinâmica GeoGebra. Foram entrevistados dez sujeitos de 14 a 17 anos, estudantes do $9^{\circ}$ ano do ensino fundamental ao $3^{\circ}$ ano do ensino médio.

Segundo Delval (2002) uma pesquisa que utiliza o Método Clínico deve seguir os seguintes passos: a escolha de um problema, o estudo dos antecedentes sobre o assunto, o procedimento para a produção dos dados, a coleta dos dados através da entrevista clínica, a análise desses dados e a elaboração de um informe com as reflexões sobre os resultados encontrados.

Essa pesquisa tem como problema o estudo do pensamento dos estudantes em relação às transformações geométricas do plano, o conhecimento das suas características e propriedades, e ainda a possibilidade do uso de recursos digitais como instrumento de uma entrevista clínica. O estudo dos antecedentes sobre os assuntos a serem abordados nesta pesquisa encontram-se nas seções 2 a 4 que partem do uso das tecnologias para o pensar matemática, a metodologia do Método Clínico e as implicações das transformações geométricas para o pensamento matemático.

Para o procedimento da produção dos dados foram criadas sete situações explorando as transformações geométricas: reflexão em relação a uma reta, reflexão em relação a um ponto e a translação por um vetor. As seis primeiras situações foram criadas utilizando o GeoGebra, onde os sujeitos da pesquisa podiam manipular as figuras para reconhecer as relações geométricas encontradas pelas transformações. O uso dessa mídia digital se dá pelo fato de que proporciona a interação do objeto de estudo com os sujeitos 
da pesquisa, de onde o conhecimento matemático emerge, segundo Basso e Notare (2015) e ainda permite a visualização do pesquisador referente à ação do sujeito perante um objeto de estudo.

As três primeiras atividades (apresentadas nas Figuras 1, 2 e 3) exploraram inicialmente o que cada sujeito sabe sobre cada transformação, ou seja, o pesquisador analisa os conhecimentos já construídos, indagando sobre o que acontece com o elemento geométrico (neste caso um triângulo escaleno) diante de cada transformação: onde ficará cada vértice após a transformação, a posição do elemento transformado, seu tamanho e forma.

Com os recursos disponíveis na ferramenta tecnológica o sujeito mostra ao pesquisador como está seu pensamento sobre o conhecimento estudado construindo o objeto resultante. Além disso, o pesquisador explora o pensamento do sujeito solicitando que explique em palavras ou gestos o seu raciocínio, como mostra a imagem capturada de uma das entrevistas realizadas (Figura 4).

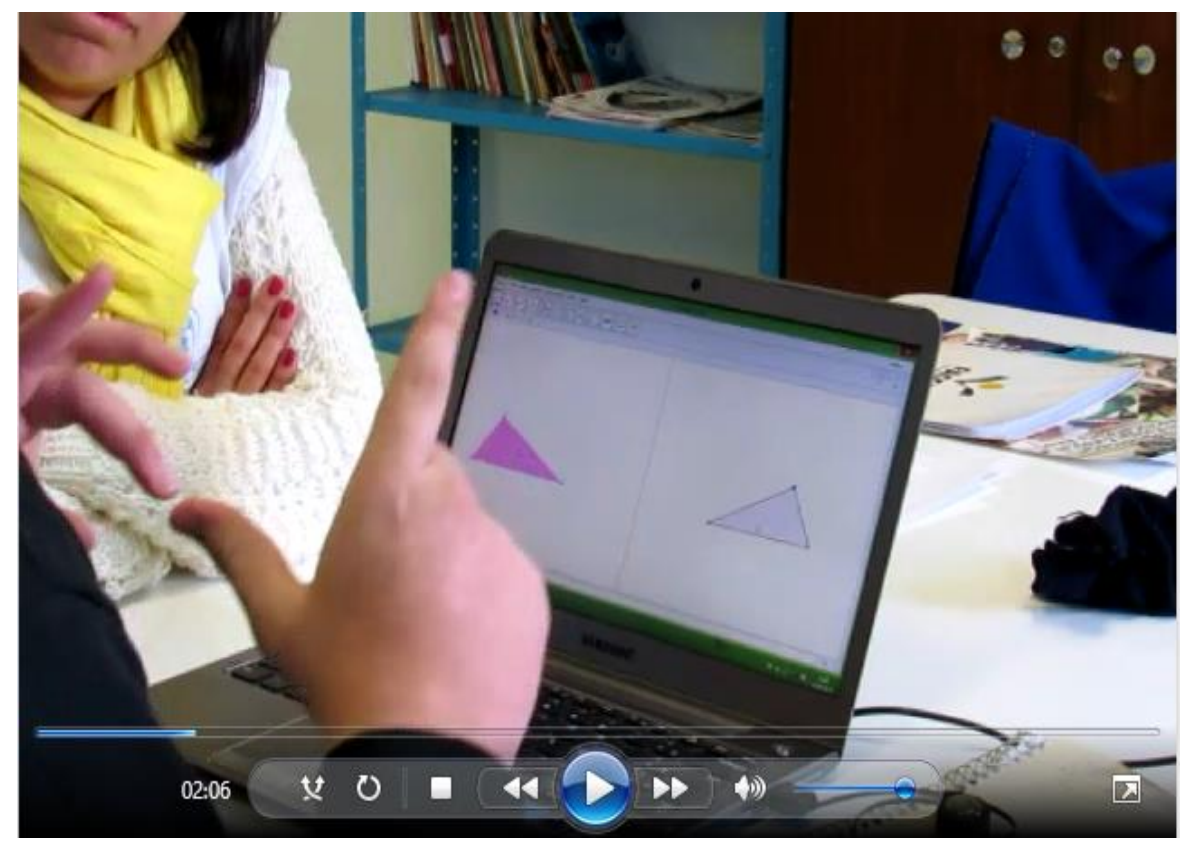

Figura 4. Exemplo da entrevista clínica

Como podemos observar na Figura 4, o pesquisador possui para a sua análise clínica a construção do objeto realizado pelo sujeito na mídia digital, como também a explicação do pensamento do sujeito através da linguagem e de gestos, pois o entrevistador realizou diferentes intervenções para conseguir analisar o pensamento do entrevistado, como sugere o Método Clínico.

Após os sujeitos analisados criarem e explicarem seu pensamento sobre cada transformação, o recurso tecnológico proporcionava ao entrevistado a comprovação do seu pensamento, pois o recurso utilizado GeoGebra dispõe da ferramenta das transformações, ou seja, o sujeito após explorar seus conhecimentos sobre o assunto tinha a oportunidade de verificar se seu pensamento estava correto. Esse momento da entrevista parte da ideia da pesquisadora que a entrevista clínica também pode ser um instrumento de aprendizagem, o sujeito é provocado em relação a certo conhecimento, explora suas operações já formadas e no final ele tem a oportunidade de verificar se suas operações estão corretas ou acaba adquirindo a experiência de algo novo. O sujeito tem a disposição no software a manipulação para verificar e explorar as características e propriedades que 
cada transformação realizou, mudando o formato da figura original, o tamanho e a posição dos elementos envolvidos.

Para o pesquisador certificar-se das respostas do sujeito e do percurso do seu pensamento em relação às transformações geométricas, as três próximas atividades propõem elementos geométricos com as transformações já realizadas, como mostra a Figura 5. O sujeito deve manipular os objetos, mudando seu tamanho, posição para se certificar da transformação realizada.

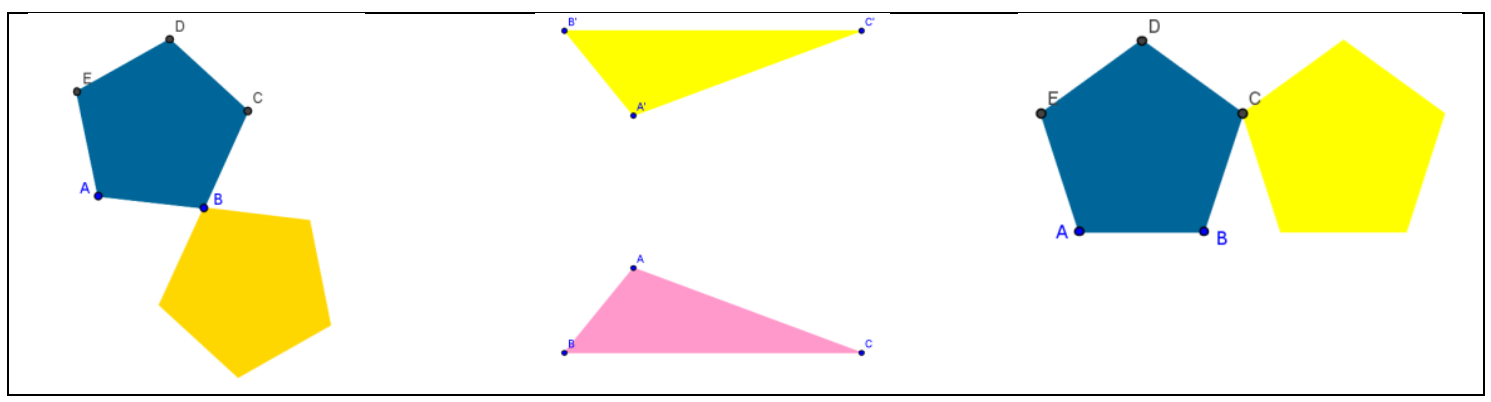

Figura 5. Atividades 4, 5 e 6 da entrevista clínica

A última situação proposta aos sujeitos da pesquisa trata da imagem de um mosaico (Figura 6), na qual aparecem diversas transformações geométricas. Para analisar o pensamento dos estudantes, o pesquisador solicita que examinem a imagem e descrevam onde se encontram e quais são as transformações vistas anteriormente.

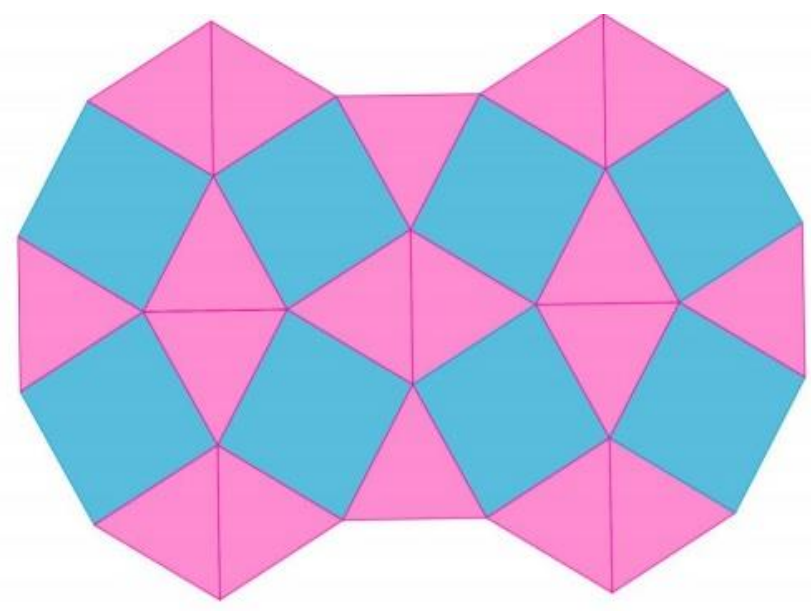

Figura 6. Mosaico utilizado na atividade 7 da entrevista clínica

A seguir serão apresentadas as análises dos resultados obtidos na entrevista clínica realizada. Para essa análise a pesquisadora baseou-se nas suas lembranças do momento da entrevista, como também, cada entrevista foi gravada em vídeo mostrando as atividades dos sujeitos sobre os objetos de estudo, ou seja, suas manipulações no software, como também suas falas e gestos. Para isso, a autora possui os termos de consentimento e assentimento dos sujeitos.

\section{Resultados preliminares}

Em relação aos resultados obtidos verificou-se que os sujeitos entrevistados possuem conhecimento sobre a reflexão em relação à reta, devido à sua experiência com simetria por espelhamento, os alunos fizeram a reflexão do objeto e explicaram como a figura ficaria após a reflexão. Alguns dos entrevistados não se preocuparam com a 
posição horizontal da reflexão, alegando que não levaram isso em consideração, mas quando a pesquisadora fez intervenções em relação à mudança do objeto inicial ou da reta, os sujeitos entrevistados responderam corretamente sobre a mudança da posição do objeto refletido.

Sobre a reflexão em relação ao ponto nenhum dos sujeitos mostrou ter essa operação interiorizada, o pesquisador ainda considera que devido a experiência anterior da reflexão em relação à reta, os sujeitos entrevistados trouxeram aquele conhecimento discutido anteriormente para este, pois todos fizeram a reflexão como na reta. Como o software disponibiliza esse recurso, os entrevistados puderam explorar o resultado dessa transformação, assim o pesquisador desafiou os sujeitos novamente a fazerem o resultado da mesma transformação, mas utilizando outro ponto. Isso é esperado no Método Clínico, o entrevistador deve estar preparado para intervenções e mudanças nas perguntas conforme a conduta dos sujeitos (Delval, 2002). Nesse momento, o recurso do software foi utilizado para explorar e realizar uma nova conjectura, promovendo o pensamento geométrico a partir de uma nova experiência (Basso; Notare, 2015).

A terceira transformação explorada teve o empecilho de alguns sujeitos desconhecerem a definição de vetor e, nessa situação, o entrevistador interferiu informando esse conceito. Alguns sujeitos realizaram a translação corretamente, como mostra a Figura 7, consideram a direção, sentido e comprimento do vetor para transladar o triângulo (o triângulo vazado é o construído pelo sujeito e A'B'C' é o triângulo realizado pela ferramenta de translação do software).
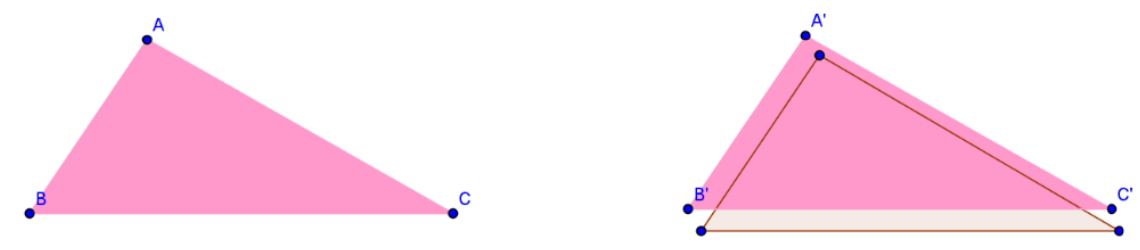

Figura 7. Construção de um sujeito em relação à translação

Mas outros sujeitos ainda não têm esse conhecimento totalmente estruturado, pois consideraram o tamanho do vetor como a distância entre os dois triângulos, o original e transladado, como mostramos na Figura 8; aqui observa-se que não transladaram cada vértice do triângulo de acordo com esse comprimento como se pode identificar pela imagem do triângulo mais claro construído pelo aluno.
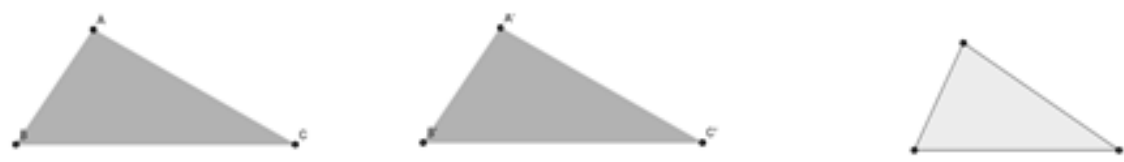

Figura 8. Construção equivocada de um sujeito em relação à translação 


\section{Conclusões finais}

Após a entrevista realizada constatou-se a complexidade da atividade de análise do pensamento de um sujeito, bem como o desafio de criar situações que favoreçam o raciocínio. Constatou-se também a necessidade de formular perguntas corretas e não sugeridas de maneira que os sujeitos expressem seu pensamento de maneira espontânea. Essas considerações nos levam a refletir que aplicar o Método Clínico requer um estudo profundo antecipado e a experiência em entrevistar clinicamente, como menciona Delval (2002).

Contudo, as construções realizadas no software facilitaram a análise desse pensamento, pois constatamos que somente em palavras o sujeito não conseguia expressar seus conhecimentos e, via uso do recurso digital, ele tinha a oportunidade de mostrar como pensava, pois, manipulava o software de acordo com seu pensamento. Ressaltamos ainda, que os sujeitos dessa pesquisa já tinham conhecimento sobre recursos do software utilizado e, nesse caso, seu uso não interferiu de maneira a impedir o seu uso para operar com os conceitos estudados.

Percebemos ainda que em algumas situações, a manipulação do sujeito sobre os elementos geométricos disponibilizados no software fez com que ele construísse novos conhecimentos, pois o momento da entrevista lhe proporcionou uma experiência de um novo conhecimento.

Essa experiência nos proporcionou reflexões sobre o uso de recursos tecnológicos para entender o pensamento dos indivíduos através da entrevista clínica. As análises aqui apresentadas não apresentam o pensamento de cada indivíduo entrevistado, mas sim, evidencia como o Método Clínico pode ser utilizado com as tecnologias digitais. Na continuidade e aprofundamento do estudo, pretende-se explorar novas potencialidades das tecnologias digitais na construção de conceitos matemáticos, pensamento geométrico espacial, utilizando como uma proposta de método para compreender esse desenvolvimento cognitivo as ideias do Método Clínico.

\section{Referências}

BASSO, Marcus; NOTARE, Márcia Rodrigues. Pensar-com Tecnologias Digitais de Matemática Dinâmica. Renote, Porto Alegre, v. 13, n. 2, 2015.

BRASIL. Parâmetros Curriculares Nacionais: terceiro e quarto ciclos do ensino fundamental - Matemática. Brasília: MEC, 1998.

2017.

Base Nacional Comum Curricular. Brasília: Secretaria de Educação Básica,

DELVAL, Juan. Introdução à Prática do Método Clínico: descobrindo o pensamento das crianças. Trad. Fátima Murad. Porto Alegre: Artmed, 2002.

GRAVINA, Maria Alice. Geometria Dinâmica: uma nova abordagem para o aprendizado da geometria. In: Anais do VII Simpósio Brasileiro de Informática na Educação, Belo Horizonte, nov. 1996, p. 1-13.

GRAVINA, Maria Alice; BASSO, Marcus Vinicius de Azevedo. Mídias digitas na Educação Matemática. In: GRAVINA, Maria Alice et al. (Org). Matemática, Mídias Digitais e Didática: tripé para formação de professor de Matemática. Porto Alegre: Evangraf, 2012. 
MEDEIROS, Margarete Farias. Geometria dinâmica no ensino de transformações no plano: uma experiência com professores da educação básica. Porto Alegre: UFRGS, 2012. 172 f. Dissertação (Mestrado em Ensino de Matemática) - Programa de PósGraduação em Ensino de Matemática, Instituto de Matemática, Universidade Federal do Rio Grande do Sul, Porto Alegre, 2012.

STORMOWSKI, Vandoir. Estudando matrizes a partir das transformações geométricas. Porto Alegre: UFRGS, 2008. 157 f. Dissertação (Mestrado em Ensino de Matemática) - Programa de Pós-Graduação em Ensino de Matemática, Instituto de Matemática, Universidade Federal do Rio Grande do Sul, Porto Alegre, 2008.

TALL, David. Information Technology and Mathematics Education: Enthusiasms, Possibilities \& $\quad 1998 . \quad$ Realities. Disponível em: <http://www.warwick.ac.uk/staff/David.Tall/downloads.html>. Acesso em: 16/03/2015. 